\title{
Using multifractal detrended fluctuation analysis to assess sacral skin blood flow oscillations in people with spinal cord injury
}

\author{
Fuyuan Liao, PhD; Yih-Kuen Jan, PT, PhD* \\ Department of Rehabilitation Sciences, University of Oklahoma Health Sciences Center, Oklahoma City, OK
}

\begin{abstract}
The purpose of this study was to investigate whether the multifractal detrended fluctuation analysis (MDFA) of skin blood flow oscillations (BFO) differed between nondisabled controls and people with spinal cord injury (SCI). The study of skin BFO has shown promise for assessing blood flow control mechanisms and risk for pressure ulcers. We recruited 23 subjects, including 11 people with SCI and 12 nondisabled controls. Thermally induced maximal sacral skin BFO were measured by laser Doppler flowmetry. MDFA was used to characterize nonlinear complexity of metabolic $(0.0095$ to $0.02 \mathrm{~Hz})$, neurogenic $(0.02$ to $0.05 \mathrm{~Hz})$, and myogenic $(0.05$ to $0.15 \mathrm{~Hz})$ BFO. We found that maximal vasodilation was significantly smaller in people with SCI than in nondisabled controls $(p<0.05)$. MDFA showed that metabolic $\mathrm{BFO}$ exhibited less complexity in people with $\mathrm{SCI}(p<$ $0.05)$, neurogenic $\mathrm{BFO}$ exhibited less complexity in people with complete SCI $(p<0.05)$, and myogenic BFO did not show significant differences between people with SCI and nondisabled controls. This study demonstrated the feasibility of using the MDFA to characterize nonlinear complexity of BFO, which is related to vasodilatory functions in people with SCI.
\end{abstract}

Key words: blood flow oscillations, detrended fluctuation analysis, laser Doppler, microvascular function, multifractal analysis, nonlinear analysis, pressure ulcers, spectral analysis, spinal cord injury, vasodilatory function.

\section{INTRODUCTION}

Spinal cord injury (SCI) causes interruption of the autonomic pathways from the brain stem and hypothalamus to the intermediolateral cell column of the spinal cord [1]. This interruption results in a loss or attenuation of modulation of the spinal autonomic reflexes in response to various stimuli at the level below spinal injury. SCI also causes immobility with a subsequent consequence of prolonged sitting and bed rest. Assuming these postures for a long time not only causes a reduction of blood flow to the compressed soft tissues but also results in an increase of skin temperature [2]. Such an increase raises the metabolic demands of local cells, which may further aggravate the tissue viability of weight-bearing tissues in people with SCI. In nondisabled individuals, an increased temperature elicits a vasodilatory response to remove excessive heat. People with SCI may not be able to induce effective vasodilation under a thermal stress. The impaired thermoregulatory function contributes to the development of pressure ulcers in people with SCI [3]. In addition to the temperature factor, other causative factors of pressure ulcers include pressure, shear, and moisture [2].

Abbreviations: $\mathrm{AV}=$ arteriovenous, $\mathrm{BFO}=$ blood flow oscillations, $\mathrm{BTI}=$ biphasic thermal index, $\mathrm{DFA}=$ detrended fluctuation analysis, eNOS = endothelial nitric oxide synthase, $\mathrm{LDF}=$ laser Doppler flowmetry, MDFA $=$ multifractal DFA, NS = nonsignificant, $\mathrm{SCI}=$ spinal cord injury.

* Address all correspondence to Yih-Kuen Jan, PT, PhD; Department of Rehabilitation Sciences, University of Oklahoma Health Sciences Center, 1200 North Stonewall Avenue, Room 3135, Oklahoma City, OK 73117; 405-271-2131, ext 47128; fax: 405-271-2432. Email: vian@ouhsc.edu DOI:10.1682/JRRD.2010.08.0145 
Skin blood flow signals contain five periodic components [4-6]. A periodic oscillation with a characteristic frequency around $0.01 \mathrm{~Hz}$ is reported to be associated with metabolic activity [5,7]. Other underlying physiological mechanisms include neurogenic $(0.02-0.05 \mathrm{~Hz})$, myogenic $(0.05-0.15 \mathrm{~Hz})$, respiratory $(0.15-0.40 \mathrm{~Hz})$, and cardiac $(0.4-2.0 \mathrm{~Hz})$ origins. However, the characteristic frequencies embedded in blood flow oscillations (BFO) show a time-varying feature that cannot be fully characterized by linear methods, such as wavelet and Fourier transforms [8-10]. This time-varying feature of characteristic frequencies may be due to the interactions of each control mechanism [7-9]. Stefanovska et al. proposed a model consisting of linear and nonlinear oscillators to understand this phenomenon [8]. Based on this model, the variations in frequency and amplitude of a periodic component are due to the interactions among the control mechanisms of BFO. The nonlinear properties of skin BFO were also reported by Liao et al. and Humeau et al. [11-12]. These studies showed that nonlinear properties of BFO may be related to vasodilatory function: a lower nonlinear complexity of BFO is associated with a smaller vasodilatory response.

Recently, nonlinear complex properties of BFO were quantified with the use of detrended fluctuation analysis (DFA) [13] by Esen's group [14-15]. They showed that a distinct scaling region, called local region, of the DFA is directly related to the metabolic, neurogenic, and myogenic mechanisms. Furthermore, the scaling exponents in the local region are from only the local origins and are not influenced by systemic factors [15]. This method also allows researchers to detect microvascular dysfunction based on the baseline blood flow without evoking the microvascular response. (The traditional method needs to induce a stimulus into the microcirculatory system to assess its response for assessing microvascular dysfunction.) However, skin blood flow signals have been shown to have multifractal behavior [16-17]. Whether these nonlinear properties of BFO are associated with specific physiological meanings is unclear.

We have conducted a series of studies correlating pathological conditions to the changes of BFO in order to investigate the role of microvascular dysfunction in pressure ulcer risk $[7,9,18]$. In our previous studies, we successfully isolated physiological mechanisms by using (linear) wavelet-based spectrum analysis of BFO [6-7]. Our method has shown promise for further quantifying the influences of pathological conditions (e.g., SCI, aging, and diabetes mellitus) on microvascular functions, which may lead to a better understanding of the development of pressure ulcers. To achieve our long-term goal of predicting pressure ulcer risk based on microvascular functions, we examined the effect of SCI on the nonlinear complexity of BFO in this study. The specific aims were to investigate the changes of nonlinear complexity of the metabolic $(0.0095-0.02 \mathrm{~Hz})$, neurogenic $(0.02-0.05 \mathrm{~Hz})$, and myogenic $(0.05-0.15 \mathrm{~Hz}) \mathrm{BFO}$ by using multifractal DFA (MDFA).

\section{METHODS}

\section{Participants}

We recruited 23 subjects, including 11 people with SCI and 12 nondisabled controls. The inclusion criteria for the SCI participants included an injury level between cervical 4 and thoracic 12, nonambulation (wheelchair users), and time after spinal injury of 6 months. None of the participants had cardiorespiratory disease, hypertension, diabetes mellitus, or other pathological conditions or were taking medications that might affect cardiovascular function. People with SCI were recruited from the University of Oklahoma Health Sciences Center and University of Central Oklahoma Wellness Center. The demographic features of the enrolled subjects are shown in the Table.

Table.

Subjects demographics. Data are expressed as median (lower quartile, upper quartile), unless otherwise indicated.

\begin{tabular}{lccc}
\hline \multicolumn{1}{c}{ Demographic } & Complete SCI & Incomplete SCI & Nondisabled Controls \\
\hline No. of Subjects & 4 & 7 & 12 \\
Sex: $\mathrm{M} / \mathrm{F}$ & $3 / 1$ & $4 / 3$ & $4 / 8$ \\
Age $(\mathrm{yr})$ & $33.0(27,46)$ & $43.0(34,49)$ & $24.0(22,26)$ \\
Duration of SCI $(\mathrm{yr})$ & $7.0(4.6,10.5)$ & $5.7(2.4,10.6)$ & - \\
Body Mass Index $\left(\mathrm{kg} / \mathrm{m}^{2}\right)$ & $24.1(22.2,25.2)$ & $24.1(22.0,28.1)$ & $23.6(21.0,25.8)$ \\
\hline
\end{tabular}

$\mathrm{F}=$ female, $\mathrm{M}=$ male, $\mathrm{SCI}=$ spinal cord injury. 


\section{Procedures}

After at least $30 \mathrm{~min}$ of quiet rest to become acclimated to the room temperature $\left(24{ }^{\circ} \mathrm{C} \pm 2{ }^{\circ} \mathrm{C}\right)$, the participant was positioned in a prone posture. Sacral skin $\mathrm{BFO}$ were recorded at a sampling rate of $32 \mathrm{~Hz}$ with laser Doppler flowmetry (LDF) (PF 5001, Perimed AB; Järfälla, Sweden). A heating probe (Probe 415-242, Perimed $\mathrm{AB}$ ) was used to heat the skin to $42{ }^{\circ} \mathrm{C}$ in $2 \mathrm{~min}$ and maintain that temperature for the duration of the $50 \mathrm{~min}$ heating period. The protocol included a $10 \mathrm{~min}$ preheating period, a $50 \mathrm{~min}$ heating period, and a $10 \mathrm{~min}$ postheating period [19]. The LDF uses a $780 \mathrm{~nm}$ laser diode and a fiber separation (distance between the transmitting and receiving fibers) of $0.25 \mathrm{~mm}$. The configuration leads to a measurement depth on the order of $1 \mathrm{~mm}$ [20].

\section{Biphasic Thermal Index}

To create a reference benchmark, we used the biphasic thermal index (BTI) [7] to incorporate the information of biphasic vasodilatory response. The index was based on Minson and colleagues' protocol in which they used a fast local heating procedure to induce a biphasic vasodilatory response [19]. The biphasic response includes two peak values of blood flow under local heating. The first peak is mediated by the activity of sensory nerves and can be modulated by the sympathetic nervous system. The second peak (plateau) is mediated by local release of endothelial nitric oxide. The BTI consists of three ratios: ratio of the first peak to baseline blood flow (P1/Baseline), ratio of the nadir to baseline blood flow (Nadir/Baseline), and ratio of the second peak to baseline blood flow (P2/Baseline). The first peak is mainly mediated by sensory axon reflex and the second peak is primarily mediated by endothelial nitric oxide [19].

\section{Multifractal Detrended Fluctuation Analysis}

A monofractal signal is homogeneous, in the sense that it has the same scaling properties throughout the entire signal [17]. A multifractal signal, on the contrary, contains many subsets that have different scaling properties. The multifractality may exhibit in several ways: a type of correlation on small (time) scales and another type of correlation or uncorrelated behavior on larger scales, different scaling behavior in different parts of the time series, and different scaling behaviors in interwoven fractal subsets of the series [17].

MDFA has been shown to reliably determine the multifractal scaling behavior of time series [17]. This method is based on a generalization of the standard DFA. Its procedure can be briefly described as follows. First, for a time series $x(k)$ of length $N$ on a compact support, one determines the integrated signal profile

$$
Y(i)=\sum_{k=1}^{i}(x(k)-<x>), i=1, \ldots, N
$$

where $\langle x\rangle$ is the mean of the time series. Then, the profile is divided into $N_{S}$ nonoverlapping segments of length $s$ and the same procedure is repeated starting from the opposite end ( $2 N_{s}$ segments total). The local trend for each segment $v$ is estimated by fitting a $m$ th order polynomial $P_{v}^{(m)}$ and subtracting it from the segment. Next, one determines the variance

$$
F^{2}(v, s)=\frac{1}{s} \sum_{i=1}^{s}\left\{Y[(v-1) s+i]-P_{v}^{(m)}(i)\right\}^{2}
$$

for each segment $v, v=1, \ldots N_{s}$ and

$$
F^{2}(v, s)=\frac{1}{s} \sum_{i=1}^{s}\left\{Y\left[N-\left(v-N_{s}\right) s+i\right]-P_{v}^{(m)}(i)\right\}^{2}
$$

for $v=N_{s}+1, \ldots, 2 N_{s}$. Finally, a $q$ th order fluctuation function is defined as

$$
F_{q}(s)=\left\{\frac{1}{2 N_{s}} \sum_{v=1}^{2 N_{s}}\left[F^{2}(v, s)\right]^{q / 2}\right\}^{1 / q}
$$

for $q \neq 0$ and $F_{0}(s)$ is defined as

$$
F_{0}(s)=\exp \left\{\frac{1}{4 N_{s}} \sum_{\mathrm{v}=1}^{2 N_{s}} \ln \left[F^{2}(v, s)\right]\right\} \quad .
$$

If the series $x(k)$ is long range power-law correlated, $F_{q}(s)$ increases for $s$ values in a certain range, as a powerlaw

$$
F_{q}(s) \sim s^{h(q)} \quad .
$$

For $q=2$, the standard DFA procedure is retrieved. The $q$ th order fluctuations $F_{q}(s)$ plotted versus various observation window sizes (scales) $s$ form a family of lines. The slopes of these lines estimate the exponents $h(q)$. Figure 1 shows an example of the relationship between the $q$ values and observation windows (scales, $s$ ). 

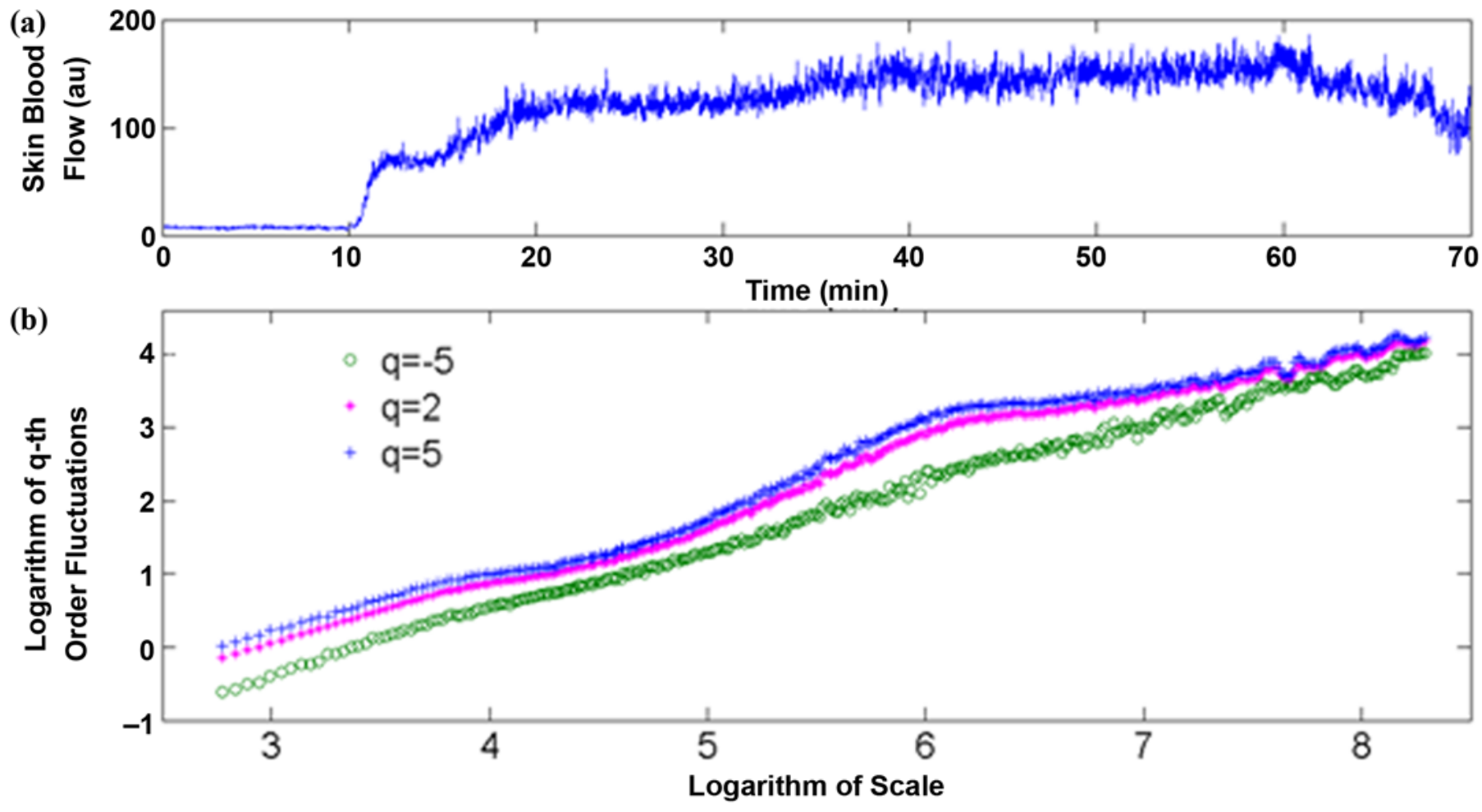

Figure 1.

Illustration of multifractal detrended fluctuation analysis of skin blood flow signal. (a) Blood flow signal was recorded on sacral skin during local heating. (b) $q$ th order fluctuations of integrated and detrended data (10 min preheating period) versus observation window sizes (scales) on log$\log$ scale, where $q \in[-5,5]$ with step 0.1 . Three curves correspond to $q=-5,2$, and 5 . Slopes of curves in certain scaling regions give estimations of exponents $h(q)$.

For a monofractal signal, $h(q)$ is independent of $q$. For a multifractal signal, small and large fluctuations scale differently, thus $h(q)$ is dependent on $q$. The reason is that for positive values of $q, h(q)$ describes the scaling behavior of the segments with large fluctuations, while for negative values of $q, h(q)$ describes the scaling behavior of the segments with small fluctuations. For stationary, normalized series, the exponents $h(q)$ are directly related to the scaling exponents $\tau(q)$, and $\tau(q)$ is defined as [17]

$$
\begin{gathered}
\tau(q)=q h(q)-1 \text { and } \\
\alpha=h(q)+q h(q) \text { and } f(\alpha)=q[\alpha-h(q)]+1,
\end{gathered}
$$

where $\alpha$ is the Hölder exponent and $f(\alpha)$ is the dimension of the subset of the time series that is characterized by $\alpha$.
In the standard DFA, the scaling exponent of a skin blood flow signal in the local region originates from only the local factors [15] and a scale $s$ is related to a frequency $f$ by $s=f_{s} / f$, where $f_{s}$ is the sampling frequency [14]. Here, we show that for an appropriate $q$ interval, the exponents $h(q)$ in the scale intervals $f_{s} / 0.02<s<f_{s} /$ 0.0095 and $f_{s} / 0.05<s<f_{s} / 0.02$ reflect the scaling properties of metabolic activity $(0.0095-0.02 \mathrm{~Hz})$ and neurogenic activity $(0.02-0.05 \mathrm{~Hz})$, respectively. As illustrated in Figure 2, after we remove the components with frequencies $0.0095<f<0.02 \mathrm{~Hz}$, scaling exponents in the region $f_{s} / 0.02<s<f_{s} / 0.0095$ are close to zero for all values of $-5 \leq q \leq 5$, while the scaling exponents in other scaling regions are identical to that of the original data. The process applies to the neurogenic BFO $(0.02<$ $f<0.05 \mathrm{~Hz}$ ) (Figure 3). This evidence indicates that for $-5 \leq q \leq 5$, the scaling exponents of a blood flow signal in the interval $f_{s} / 0.02<s<f_{s} / 0.0095$ and $f_{s} / 0.05<s<f_{s} / 0.02$ reflect the scaling behavior of metabolic and neurogenic 


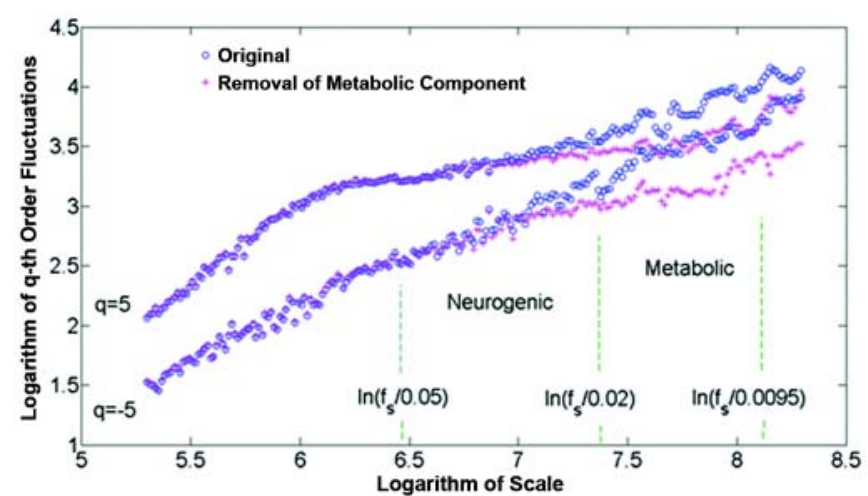

Figure 2.

Illustration of scale interval related to metabolic frequency $(0.0095-$ $0.02 \mathrm{~Hz}$ ) of skin blood flow oscillations. For baseline blood flow signal shown in Figure 1(a), after we removed metabolic component, scaling exponents (slopes of lines) in scale interval $f_{s} / 0.02<s<f_{s} / 0.0095$ are close to zero, while scaling exponents in other scaling regions are nearly identical to original signal, where $f_{s}$ is sampling frequency. Because components in frequency band $0.0095-0.02 \mathrm{~Hz}$ cannot be completely removed and removed components are not perfectly in frequency band $0.0095-0.02 \mathrm{~Hz}$, scaling exponents in $f_{s} / 0.02<s<$ $f_{s} / 0.0095$ are nonzero and in other scale intervals are not exactly identical to original signal, especially for $q<0$.

$\mathrm{BFO}$, respectively. The same procedures can be used to process the myogenic BFO.

Previous studies have used the range of Hölder exponent [21-22] or $h\left(q_{\min }\right)-h\left(q_{\max }\right)$ [23] to quantify the multifractality degree of time series. However, estimates of the range of Hölder exponent often encounter a problem of numerical instability [24]. The results of simulation experiments showed that the range of $h(q), \Delta h=$ $\max (h(q))-\min (h(q))$ is more robust than $h\left(q_{\text {min }}\right)-$ $h\left(q_{\max }\right)$ for typical monofractal signals, e.g., fractional Brownian motions $(\mathrm{fBmH}, \mathrm{H}$ is the Hurst exponent, $0<$ $\mathrm{H}<1$ ). On the other hand, for $\mathrm{fBmH}$ with the length of 10 min blood flow data, values of $\Delta h$ in the scale intervals $f_{s} / 0.02<s<f_{s} / 0.0095$ and $f_{s} / 0.05<s<f_{s} / 0.02$ are significantly smaller than that of blood flow data, especially in nondisabled people, indicating nondisabled people exhibit multifractality in BFO. Therefore, we used $\Delta h=\max (h(q))-\min (h(q))$ in the scale interval $f_{s} / 0.02<$ $s<f_{S} / 0.0095$ to quantify the multifractality degree of metabolic BFO, the scale interval $f_{s} / 0.05<s<f_{s} / 0.02$ to quantify the multifractality degree of neurogenic BFO, and the scale interval $f_{s} / 0.15<s<f_{s} / 0.05$ to quantify the multifractality degree of myogenic BFO.

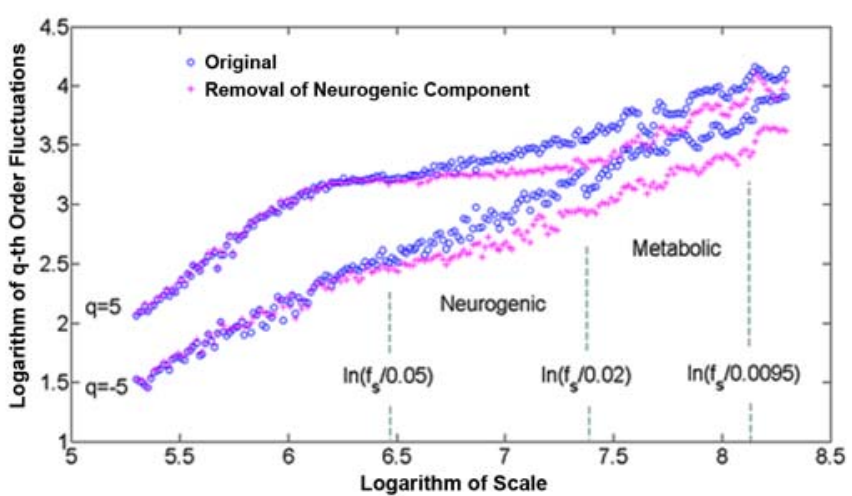

Figure 3.

Illustration of scaling interval related to neurogenic frequency band $(0.02-0.05 \mathrm{~Hz})$ of skin blood flow oscillations. For baseline blood flow signal shown in Figure 1(a), after we removed neurogenic component, scaling exponents (slopes of lines) in scale region $f_{s} / 0.05<$ $s<f_{s} / 0.02$ are close to zero, while scaling exponents in other scaling regions are nearly identical to original signal, where $f_{s}$ is sampling frequency. Because components in frequency band $0.02-0.05 \mathrm{~Hz}$ cannot be completely removed and removed components are not perfectly in frequency band $0.02-0.05 \mathrm{~Hz}$, scaling exponents in $f_{s} / 0.05<$ $s<f_{s} / 0.02$ are nonzero and in other scale intervals are not exactly identical to original signal, especially for $q<0$.

\section{Statistical Analysis}

Nonparametric statistics were used because of the small sample size. Comparisons among groups in the same thermal condition were performed using the Kruskal-Wallis tests. When the factor was significant, we further checked the significance between groups by using the Wilcoxon rank sum tests. Comparisons between two thermal conditions in the same group were performed using the Wilcoxon signed rank tests. The significance level was set at $p<0.05$. MATLAB and Signal Processing and Statistical Analysis Toolboxes (R2008b, MathWorks; Natick, Massachusetts) were used to implement MDFA and statistical testing.

\section{RESULTS}

People with SCI showed a smaller vasodilatory response than nondisabled controls (Figure 4). During the axon reflex mediated vasodilation (P1/Baseline), a significantly smaller increase in sacral skin perfusion was observed in people with complete $(p<0.01)$ and incomplete $(p<0.05)$ SCI than in nondisabled controls. During 


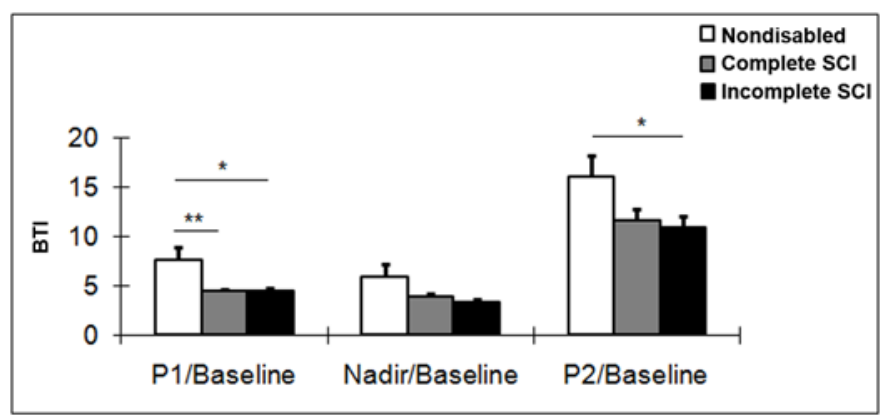

Figure 4.

Biphasic thermal index (BTI) of nondisabled controls and people with spinal cord injury (SCI) (complete and incomplete). During axon reflex mediated vasodilation (P1/Baseline), significantly smaller increase in sacral skin perfusion is observed in people with complete $(p<0.01)$ and incomplete $(p<0.05)$ SCI compared with nondisabled subjects. During maximal vasodilation (P2/Baseline), significantly smaller increase in sacral skin perfusion is observed in people with incomplete SCI compared with nondisabled subjects $(p<0.05)$. Values are expressed as mean \pm standard error. * indicates $p<0.05$ and $* *$ indicates $p<0.01$. $\mathrm{P} 1=$ first peak of biphasic vasodilation, $\mathrm{P} 2=$ second peak of biphasic vasodilation.

the maximal vasodilation (P2/Baseline), a significantly smaller increase in sacral skin perfusion was observed in people with incomplete SCI than in nondisabled controls $(p<0.05)$.

Figure 5 shows the multifractality degree $(\Delta h)$ of metabolic oscillations. In responding to local heating, the multifractality degree of metabolic oscillations significantly increased in nondisabled people $(p<0.01)$ but not in people with complete or incomplete SCI $(p>0.05$, nonsignificant $[\mathrm{NS}])$. By comparing the multifractality degree of metabolic oscillations during the maximal vasodilation, we found that people with complete or incomplete SCI showed a significantly lower value than nondisabled controls $(p<0.05)$. No statistically significant difference was found between people with complete and incomplete SCI (NS).

Figure 6 shows the multifractality degree $(\Delta h)$ of neurogenic oscillations. In responding to local heating, the multifractality degree of neurogenic oscillations significantly decreased in people with complete SCI $(p<$ $0.05)$ and people with incomplete SCI $(p<0.05)$. By comparing the multifractality degree of neurogenic oscillations during the maximal vasodilation, we found that people with complete SCI showed a significantly lower value than nondisabled controls $(p<0.05)$.

Figure 7 shows the multifractality degree $(\Delta h)$ of myogenic oscillations. No significant differences were

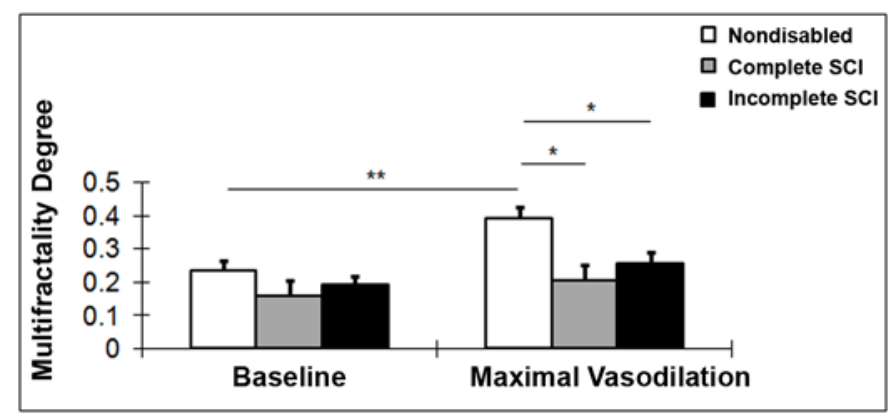

Figure 5.

Comparison of multifractality degree $(\Delta h)$ of metabolic oscillations in nondisabled controls and people with spinal cord injury (SCI) (complete and incomplete). In responding to local heating, multifractality degree of metabolic oscillations significantly increases in nondisabled subjects $(p<0.01)$ but not in people with complete or incomplete SCI. If multifractality degree of metabolic oscillations is compared during maximal vasodilation, people with complete or incomplete SCI show significantly lower values than nondisabled controls $(p<0.05)$. No statistically significant difference exists between people with complete and incomplete SCI $(p>0.05)$. Values are expressed as mean \pm standard error. * indicates $p<0.05$ and $* *$ indicates $p<0.01$.

found between people with SCI and nondisabled controls (NS).

\section{DISCUSSION}

We have shown that the complexity of metabolic oscillations $(0.0095-0.02 \mathrm{~Hz})$ in people with complete and incomplete SCI was significantly lower than in nondisabled controls during the maximal vasodilation. People with complete SCI exhibited a significantly lower complexity of the neurogenic oscillations $(0.02-0.05 \mathrm{~Hz})$ as compared with nondisabled controls. Our findings provide new insight into the mechanisms responsible for vasodilatory dysfunction in people with SCI. To the best of our knowledge, this is the first study to characterize the impairment of nonlinear complexity of metabolic and neurogenic oscillations in people with SCI and the results support our hypothesis that SCI causes a decrease of nonlinear complexity in sacral skin BFO. This result has advanced our understanding of physiological meanings of BFO and their roles of vasodilatory dysfunction. The finding may contribute to our long-term goal of using $\mathrm{BFO}$ patterns for the early detection of pressure ulcers in people with SCI $[7,9,18]$. 


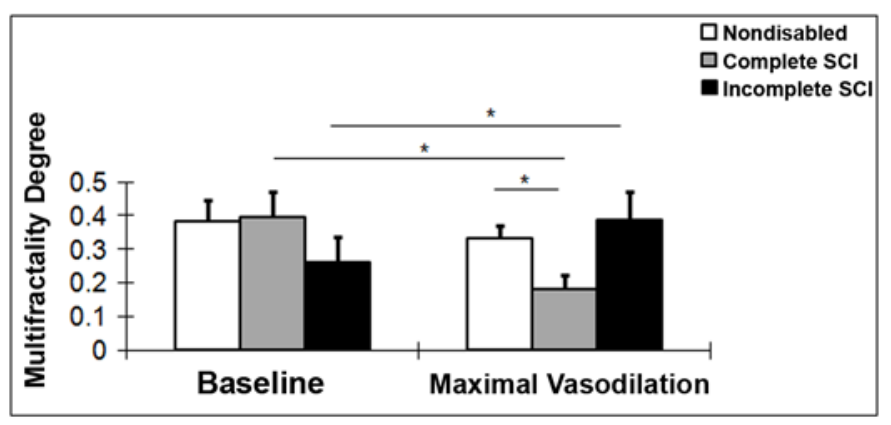

Figure 6.

Comparison of multifractality degree $(\Delta h)$ of neurogenic oscillations in nondisabled controls and people with SCI (complete and incomplete). In responding to local heating, multifractality degree of neurogenic oscillations significantly decreases in people with complete SCI $(p<$ $0.05)$ and people with incomplete SCI $(p<0.05)$. If multifractality degree of neurogenic oscillations are compared during maximal vasodilation, people with complete SCI show significantly lower values compared with nondisabled subjects $(p<0.05)$. Values are expressed as mean \pm standard error. $*$ indicates $p<0.05$.

Vasodilatory function could be used to quantify risk for pressure ulcers in people with SCI [25-27]; people with SCI who fail to increase skin blood in response to a causative factor of pressure ulcers (pressure, shear, or heating) are susceptible to pressure ulcers [27-28]. Schubert and Fagrell demonstrated that people with SCI have impaired vasodilatory response to either local heating or local pressure [27]. Hagisawa et al. showed that people with SCI take longer to reach peak hyperemia after removal of loading pressure [29]. Jan et al. showed that pressure loadings (alternating and constant pressures) induce different skin blood flow response between people with SCI and nondisabled controls [28]. Nicotra et al. observed a lower ratio of the first peak to baseline blood flow at the level below the lesion in people with complete high-level SCI (cervical 6 to thoracic 5) as compared with people with low-level SCI (thoracic 6 to 11) [26]. They suggested that this lower ratio is due to the loss of sympathetic innervations in people with high-level SCI. The need for sympathetic innervation for a full vasodilatory response was also indicated by Ping and Johnson [30]. These studies have demonstrated that people with SCI have impaired vasodilatory response to loading pressure and thermal stress. In the literature, the influences of loading pressure have drawn more attention than other factors (temperature, shear, and moisture). We are particularly interested in assessing the thermoregulatory functions and the role of local skin temperature changes due

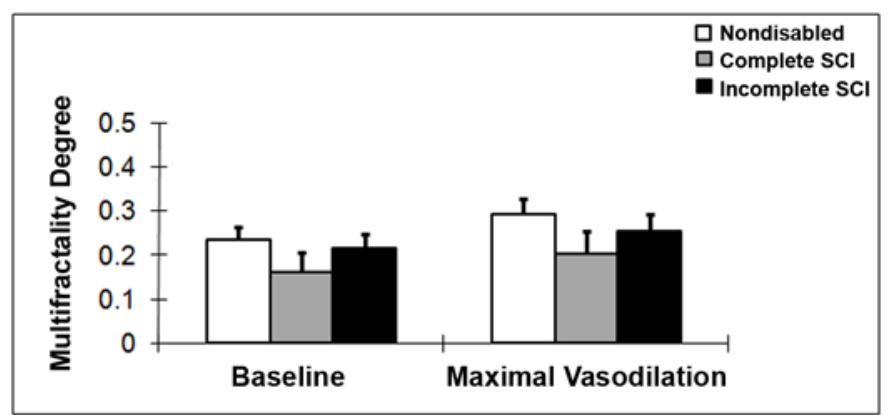

Figure 7.

Comparison of multifractality degree $(\Delta h)$ of myogenic oscillations in nondisabled controls and people with spinal cord injury (SCI) (complete and incomplete). No significant differences exist between people with SCI and nondisabled controls. Values are expressed as mean \pm standard error.

to prolonged seating in the development of pressure ulcers. In this study, we continued our research on sacral skin $\mathrm{BFO}$ and their relationship with vasoregulatory functions [6-7].

The LDF is an indirect measure of red blood cell velocity [31]. Previous studies have shown that the LDF signal is independent of the effect of the absorption characteristics of the blood for a given LDF system as the fiber separation and wavelength are fixed [32-34]. Shepherd et al. reported that laser Doppler velocity signals are unaffected by changes in blood oxygenation [32]. Nilsson et al. evaluated skin blood flow measurements based on the laser Doppler principle and found a linear relationship between flowmeter response and flux of red blood cells [33]. Fredriksson et al. evaluated the effects of blood concentration and blood oxygen saturation on the measurement depth and volume in LDF through Monte Carlo simulations of tissue using relevant computer models [34]. The results showed that, for the wavelength of $780 \mathrm{~nm}$, the effect of the oxygenation status of the blood on the measurement depth is very small.

The depth of tissue penetrated by the laser depends on factors such as tissue properties, light source wavelength, and probe configuration [20,35]. The LDF used in this study uses a $780 \mathrm{~nm}$ laser diode and the fiber separation is $0.25 \mathrm{~mm}$. This configuration leads to a measurement depth on the order of $1 \mathrm{~mm}$ for human skin [20]. The measurement depth of the LDF reaches to the arterioles, capillaries, and postcapillary venules of the upper horizontal plexus but does not extend to the deep horizontal plexus [36]. Thus, skin perfusion as measured in 
this study includes blood flow in the capillaries, arterioles, venules, and arteriovenous (AV) anastomoses (or shunts) [20]. Blood flow in the capillaries provides nutrients and oxygen to local cells, and blood flow in the arterioles, venules, and AV shunts regulate body temperature [20].

BFO have been assessed with use of the amplitudes or power in several frequency bands [5,7,37]. It has been demonstrated that attenuated metabolic and neurogenic oscillations are associated with vasodilatory dysfunction [7,37]. Li et al. observed significantly lower blood flow in people with SCI than in nondisabled controls [37]. They also showed that the amplitudes of metabolic and neurogenic frequencies are smaller in people with SCI than in nondisabled controls. However, the dynamics of skin blood flow or of a frequency component contain not only amplitude information but also structural information (nonlinear complexity). The latter cannot be characterized by the averaged amplitude in a time interval. Although wavelet-based time-frequency-amplitude presentation can describe how frequency and amplitude change with time, difficulties may arise from quantifying the changes [7,9]. MDFA allows us to quantify the dynamics of a frequency component with respect to its complexity. From our results, we may deduce that metabolic oscillations in nondisabled people have richer structures (i.e., more complex behavior) than in people with SCI. Our results support the general concept that the output of a healthy system reveals complex variability and that the complexity decreases with aging and diseases [16,38-39]. Reduced complexity of the physiological system is widely accepted to be associated with an individual's poor abilities to adapt to environmental stimulus. Such vasodilatory impairment may increase risk for tissue ischemic damage [7].

Variations in the frequency and amplitude of the metabolic oscillations are likely induced by interactions between other control mechanisms (e.g., neurogenic and myogenic) of skin microcirculation. The cardiovascular system has been modeled as coupled linear and nonlinear oscillators to study the time-varying features of characteristic frequencies [8]. Metabolic activity adjusts blood flow to meet the need of cells for oxygen. This activity is dependent on cardiac and respiratory activities, which control the level of oxygenated blood flow. On the other hand, the constrictor activities of myogenic and neurogenic systems diminish blood transport, thus lowering metabolic activity [8]. Ping and Johnson found that the myogenic response could be enhanced by sympathetic tone [30]. According to these studies, we assume that the interactions between other control mechanisms result in the variations in frequency and amplitude of metabolic oscillations. This assumption is supported by our observations, including that metabolic oscillations in nondisabled people are less homogeneous than in people with SCI both in the preheating and maximal vasodilation periods and that both in nondisabled people and people with SCI, metabolic oscillations become less homogeneous in response to local heating. In nondisabled controls, the metabolic oscillator interacts with other oscillators, including the neurogenic oscillator, in an adaptive way. A broad class of outside interactions leads to metabolic oscillations over multiple time scales. These fluctuations may superimpose, counteract, or modulate each other, resulting in complex oscillations. In people with SCI, diminished neurogenic activity may lower the interactions among these control mechanisms. The attenuated interactions result in less complex structures of metabolic oscillations. During local heating, the power of metabolic frequency has been shown to increase at the highest order, while the powers of the other characteristic frequencies also increase but at lower orders [7]. Thus, local heating may lead to changes in the intensities of the interactions among the control mechanisms, resulting in more complex structures of metabolic oscillations.

Our results indicated that people with SCI have a lower complex behavior of metabolic oscillations. The underlying mechanism of the attenuated metabolic endothelial function in people with SCI is unclear. One possible reason is their physically inactive lifestyle. The occurrence of endothelial dysfunction is primarily the result of reduced nitric oxide bioavailability [40]. Nitric oxide is a vasoactive substance that plays a pivotal role in the maintenance of vascular homeostasis. The amount of nitric oxide available depends on several key factors including the activity of the endothelial nitric oxide synthase (eNOS) and nitric oxide degradation. People with SCI are likely inactive relative to nondisabled controls. Physical activity induces increases in shear stress on the endothelium, which can lead to an increase in eNOS expression [40]. Furthermore, exercise increases phosphorylation and can also positively influence the nitric oxide half-life by reducing nitric oxide degradation. According to the literature, physical activity may slow down or even reverse metabolic dysfunction in people with SCI. MDFA of skin BFO, the method proposed in this study, could be used to assess the effectiveness of 
exercise interventions on enhancing microvascular function in people with SCI. Our speculation may be validated by examining the influence of exercise on maximal vasodilation and multifractality degree of metabolic oscillations in people with SCI. A longitudinal monitoring of skin perfusion in people with SCI who are enrolled in an exercise program might demonstrate an increase of maximal vasodilatory function and complex behavior of skin BFO. Another reason for metabolic endothelial dysfunction in the SCI population is reduced sympathetic tone. Miranda and Hassouna suggested that the interruption of neurological impulses causes metabolic changes in blood vessels that result in altered venous competence [41]. This hypothesis is supported by the findings of Houtman et al. [42], who found that sympathetic nervous system response was altered in people with SCI in comparison with nondisabled participants. This argument is also supported by the results of BTI. We observed significantly lower values of $\mathrm{P} 1 /$ Baseline and $\mathrm{P} 2 /$ Baseline in people with SCI (Figure 4). Since the first peak is modulated by the activity of sympathetic nerves [43] and the secondary peak is mediated by endothelial nitric oxide [19], we may deduce that in people with SCI, both sympathetic tone and nitric oxide release were reduced. Therefore, the attenuated metabolic endothelial function in people with SCI may be attributed to at least two factors, including reduced sympathetic tone and reduced nitric oxide release.

Using MDFA, we were able to characterize the nonlinear complexity of sacral skin BFO and detect that people with SCI had a lower complexity than nondisabled controls. Our results support the concept that decreased nonlinear behavior of a physiological system is associated with pathological states [16,38-39]. In nondisabled controls, a significant increase of the multifractality degree of metabolic BFO in response to local heating may be attributed to more metabolic activities and the interactions between other mechanisms. On the other hand, in people with SCI, a smaller change in the multifractality degree of metabolic oscillations in response to local heating may suggest the impairment of metabolic endothelial function and/or altered coupling between metabolic control and other regulatory mechanisms of skin microcirculation. More research is needed to investigate the consequence of SCI on the changes of nonlinear properties of BFO.

An important challenge in applying the MDFA to detect the complexity of a frequency component (e.g., metabolic component) of skin BFO is how to accurately determine the frequency band of this component. We found that the frequency band of the metabolic activity slightly varies among the participants and even varies from one time period to another in the same participant. These time-varying features have been indicated in our previous studies $[7,9]$. Thus, in order to accurately estimate the nonlinear multifractality of the metabolic oscillations or other physiological-related oscillations one must visually inspect, the energy spectrum of blood flow signal and the $\log \left(F_{q}(s)\right)-\log (s)$ plot to determine the frequency band.

Previous studies have reported that cigarette smoking influences microvascular function. Warner et al. showed that nicotine caused a decrease in resting and maximal skin blood flow responses to prolonged heating [44]. Argacha et al. demonstrated that passive smoking was associated with a prolonged rise of skin blood flow in response to local heating [45]. We did not exclude smokers from this study. Two smokers were enrolled in this study: one in the complete SCI group and the other in the nondisabled group. The smoker in the SCI group had been smoking about 8 to 10 cigarettes daily for about 10 years. The smoker in the nondisabled control group had been smoking about 15 to 20 cigarettes daily for 3 years. The BTI of the two smokers were $(4.3,4.2,10.9)$ and $(10.8$, $6.6,21.3)$, respectively; and the average BTI of the corresponding groups were $(4.5,3.9,11.6)$ and $(8.5,6.5,17.7)$, respectively. On the basis of the direct comparison, these two smokers had microvascular function consistent with the other subjects in each group. We anticipate that smoking had minimal influence on these two subjects' skin blood flow responses.

This study had several limitations. First, we only recruited 11 people with SCI into this pilot study. We intended to test the feasibility of using MDFA to assess microvascular dysfunction in people with SCI. Thus, we did not recruit a larger group of research participants. Future studies may consider a larger sample size to validate our results. Second, our design did not match age and sex between the nondisabled controls and people with SCI. Although sex and age might confound our results, the influences of these factors should be minimal [46]. Although the SCI groups were not age-matched to the nondisabled control group, the influence of the age factor should be minimal compared with the SCI factor. Previous studies reported that age is not correlated with skin blood flow in people between ages 17 and 60 [4647]. This evidence suggests that the effect of age/aging on microvascular function is usually documented in people over age 60 [48]. In this study, the median (lower and 

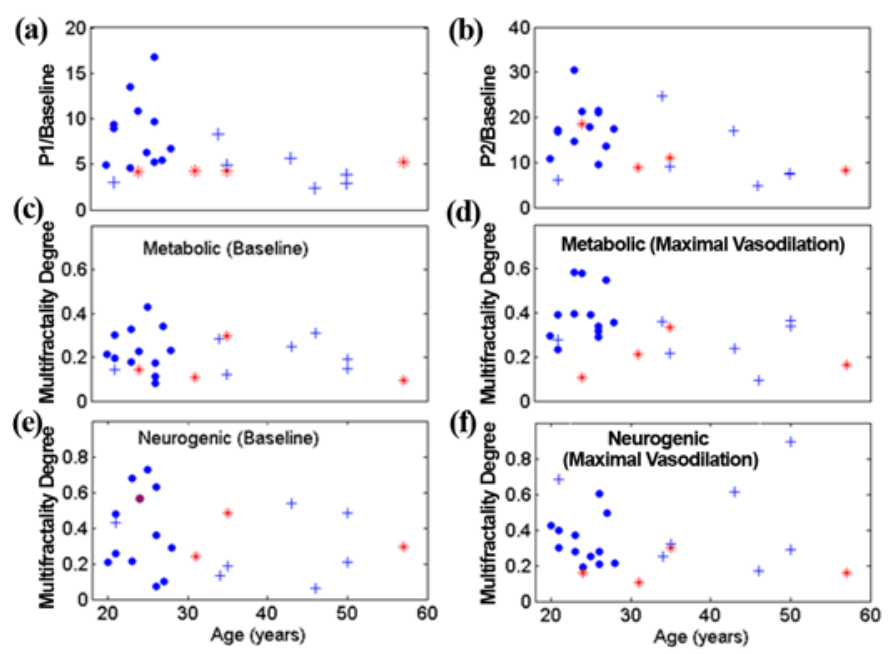

Figure 8.

Scatter plots of (a)-(b) biphasic thermal index and (c)-(f) multifractality degree of metabolic and neurogenic oscillations versus age in people with complete SCI, people with incomplete SCI, and nondisabled controls. Results indicate that no relationship exists between age and skin blood flow parameters. Filled circles represent nondisabled subjects, stars represent people with complete SCI, and pluses represent people with incomplete SCI. P1/Baseline = ratio of 1 st peak to baseline blood flow, $\mathrm{P} 2 /$ Baseline $=$ ratio of $2 \mathrm{nd}$ peak to baseline blood flow, $\mathrm{SCI}=$ spinal cord injury.

upper quartiles) ages of the complete SCI, incomplete SCI, and nondisabled groups were $33(27.5,46), 43$ (34, $49)$, and $24.5(22,26)$, respectively. Skin blood flow of these research subjects was not largely affected by age [46-47]. To assess the effect of age on skin perfusion, we performed a regression analysis between the BTI and multifractality degree and age in three subject groups. The results indicated no relationship between age and the skin blood flow-related parameters (Figure 8). Third, many other nonlinear methods (e.g., entropy, correlation dimension, Lyapunov exponent) may be used to quantify nonlinear properties of BFO in people with SCI. In this study, we only selected the MDFA to quantify complexity of BFO in people with SCI. The rationale for this selection was based on the prior publications of Esen and colleagues [14-15]. In their studies, they demonstrated the feasibility of using DFA to quantify the nonlinear properties of BFO. The feasibility of using other methods to quantify the changes of nonlinear properties of BFO in people with SCI should be examined to better understand the role of SCI in microvascular dysfunction.

\section{CONCLUSIONS}

Our results provided initial evidence that the dynamics of metabolic and neurogenic controls of skin BFO in people with SCI changed, exhibiting less complexity than those in nondisabled controls. Unlike traditional methods, in which the normalized amplitude of a frequency component is used as a quantitative measure of the related control mechanism of BFO, MDFA provides information about the nonlinear complexity of a blood flow signal, which may be used to study the interactions among various blood flow control mechanisms and the influences of SCI on microvascular functions. This method may contribute to the understanding of the effects of SCI on microvascular dysfunction and risk for pressure ulcers.

\section{ACKNOWLEDGMENTS}

\section{Author Contributions:}

Study concept and design: Y. K. Jan.

Acquisition of data: F. Liao, Y. K. Jan.

Analysis and interpretation of data: F. Liao, Y. K. Jan.

Drafting of manuscript: F. Liao, Y. K. Jan.

Critical revision of manuscript for important intellectual content:

F. Liao, Y. K. Jan.

Statistical analysis: F. Liao, Y. K. Jan.

Obtained funding: Y. K. Jan.

Administrative, technical, or material support: Y. K. Jan.

Study supervision: Y. K. Jan.

Financial Disclosures: The authors have declared that no competing interests exist.

Funding/Support: This material was based on work supported by the Paralyzed Veterans of America Research Foundation (grant PVA2480).

Additional Contributions: The authors thank Mark Anderson, PT, PhD; Shirley James, PT, MS; and Katrina Shaklee, MA, for subject recruitment. Institutional Review: All subjects gave informed consent to this study approved by the University Institutional Review Board. The nondisabled controls were university graduate students.

Participant Follow-Up: The authors plan to inform participants of the publication of this study.

Disclaimer: The views expressed in this article are those of the authors and do not necessarily reflect the position or policy of the Paralyzed Veterans of America Research Foundation.

\section{REFERENCES}

1. Alexander MS, Biering-Sorensen F, Bodner D, Brackett NL, Cardenas D, Charlifue S, Creasey G, Dietz V, Ditunno J, Donovan W, Elliot SL, Estores I, Graves DE, Green B, 
Gousse A, Jackson AB, Kennelly M, Karlsson AK, Krassioukov A, Krogh K, Linsenmeyer T, Marino R, Mathias CJ, Perkash I, Sheel AW, Schilero G, Schurch B, Sonkesen J, Stiens S, Wecht J, Wuermser LA, Wyndaele JJ. International standards to document remaining autonomic function after spinal cord injury. Spinal Cord. 2009;47(1):36-43.

[PMID: 18957962]

DOI:10.1038/sc.2008.121

2. Jan YK, Brienza DM. Tissue mechanics and blood flow factors in pressure ulcers of people with spinal cord injury. In Gefen A, editor. The pathomechanics of tissue injury and disease, and the mechanophysiology of healing. Kerala (India): Research Signpost; 2009. p. 241-59.

3. Nixon J, Cranny G, Bond S. Pathology, diagnosis, and classification of pressure ulcers: Comparing clinical and imaging techniques. Wound Repair Regen. 2005;13(4):365-72. [PMID: 16008725]

DOI:10.1111/j.1067-1927.2005.130403.x

4. Funk W, Endrich B, Messmer K, Intaglietta M. Spontaneous arteriolar vasomotion as a determinant of peripheral vascular resistance. Int J Microcirc Clin Exp. 1983;2(1):11-25. [PMID: 6678836]

5. Stefanovska A, Bracic M, Kvernmo HD. Wavelet analysis of oscillations in the peripheral blood circulation measured by laser Doppler technique. IEEE Trans Biomed Eng. 1999; 46(10):1230-39. [PMID: 10513128]

DOI:10.1109/10.790500

6. Geyer MJ, Jan YK, Brienza DM, Boninger ML. Using wavelet analysis to characterize the thermoregulatory mechanisms of sacral skin blood flow. J Rehabil Res Dev. 2004;41(6A):797-806. [PMID: 15685468]

DOI:10.1682/JRRD.2003.10.0159

7. Jan YK, Struck BD, Foreman RD, Robinson C. Wavelet analysis of sacral skin blood flow oscillations to assess soft tissue viability in older adults. Microvasc Res. 2009; 78(2):162-68. [PMID: 19465031] DOI:10.1016/j.mvr.2009.05.004

8. Stefanovska A, Lotric MB, Strle S, Haken H. The cardiovascular system as coupled oscillators? Physiol Meas. 2001; 22(3):535-50. [PMID: 11556673] DOI:10.1088/0967-3334/22/3/311

9. Jan YK, Brienza DM, Geyer MJ. Analysis of week-toweek variability in skin blood flow measurements using wavelet transforms. Clin Physiol Funct Imaging. 2005; 25(5):253-62. [PMID: 16117727] DOI:10.1111/j.1475-097X.2005.00621.X

10. Ursino M, Colantuoni A, Bertuglia S. Vasomotion and blood flow regulation in hamster skeletal muscle microcirculation: A theoretical and experimental study. Microvasc Res. 1998;56(3):233-52. [PMID: 9828162]

DOI:10.1006/mvre.1998.2106

11. Liao F, Garrison DW, Jan YK. Relationship between nonlinear properties of sacral skin blood flow oscillations and vasodilatory function in people at risk for pressure ulcers.
Microvasc Res. 2010;80(1):44-53. [PMID: 20347852]

DOI:10.1016/j.mvr.2010.03.009

12. Humeau A, Chapeau-Blondeau F, Rousseau D, Rousseau P, Trzepizur W, Abraham P. Multifractality, sample entropy, and wavelet analyses for age-related changes in the peripheral cardiovascular system: Preliminary results. Med Phys. 2008; 35(2):717-23. [PMID: 18383693] DOI:10.1118/1.2831909

13. Peng CK, Hausdorff JM, Havlin S, Mietus JE, Stanley HE, Goldberger AL. Multiple-time scales analysis of physiological time series under neural control. Physica A. 1998; 249:491-500. [PMID: 11541513] DOI:10.1016/S0378-4371(97)00508-6

14. Esen F, Aydin GS, Esen H. Detrended fluctuation analysis of laser Doppler flowmetry time series. Microvasc Res. 2009;78(3):314-18. [PMID: 19660479] DOI:10.1016/j.mvr.2009.07.005

15. Esen F, Esen H. Detrended fluctuation analysis of laser Doppler flowmetry time series: The effect of extrinsic and intrinsic factors on the fractal scaling of microvascular blood flow. Physiol Meas. 2006;27(11):1241-53. [PMID: 17028415] DOI:10.1088/0967-3334/27/11/015

16. Humeau A, Chapeau-Blondeau F, Rousseau D, Tartas M, Fromy B, Abraham P. Multifractality in the peripheral cardiovascular system from pointwise holder exponents of laser Doppler flowmetry signals. Biophys J. 2007;93(12): L59-61. [PMID: 18045964] DOI:10.1529/biophysj.107.119057

17. Kantelhardt JW, Zschiegner SA, Koscielny-Bunde E, Bunde A, Havlin S, Stanley HE. Multifractal detrended fluctuation analysis of nonstationary time series. Physica A. 2002; 316(1-4):87-114. DOI:10.1016/S0378-4371(02)01383-3

18. Jan YK, Brienza DM, Geyer MJ, Karg P. Wavelet-based spectrum analysis of sacral skin blood flow response to alternating pressure. Arch Phys Med Rehabil. 2008;89(1): 137-45. [PMID: 18164343] DOI:10.1016/j.apmr.2007.07.046

19. Minson CT, Berry LT, Joyner MJ. Nitric oxide and neurally mediated regulation of skin blood flow during local heating. J Appl Physiol. 2001;91(4):1619-26. [PMID: 11568143]

20. Humeau A, Steenbergen W, Nilsson H, Strömberg T. Laser Doppler perfusion monitoring and imaging: Novel approaches. Med Biol Eng Comput. 2007;45(5):421-35.

[PMID: 17430155] DOI:10.1007/s11517-007-0170-5

21. Ivanov PC, Amaral LA, Goldberger AL, Havlin S, Rosenblum MG, Struzik ZR, Stanley HE. Multifractality in human heartbeat dynamics. Nature. 1999;399(6735):461-65. [PMID: 10365957]

DOI: $10.1038 / 20924$

22. Meyer M, Stiedl O. Self-affine fractal variability of human heartbeat interval dynamics in health and disease. Eur $\mathrm{J}$ Appl Physiol. 2003;90(3-4):305-16. [PMID: 12942331] DOI:10.1007/s00421-003-0915-2 
23. Zunino L, Tabak BM, Figliola A, Pérez DG, Garavaglia M, Rosso OA. A multifractal approach for stock market inefficiency. Physica A. 2008;387(26):6558-66.

DOI:10.1016/j.physa.2008.08.028

24. Makowiec D, Dudkowska A, Galaska R, Rynkiewicz A, Wdowczyk-Szulc J. Monofractality in RR heart rate by multifractal tools. Acta Phys Pol B. 2009;40(5):1527-47.

25. Daniel RK, Wheatley D, Priest D. Pressure sores and paraplegia: An experimental model. Ann Plast Surg. 1985;15(1): 41-49. [PMID: 4083714] DOI:10.1097/00000637-198507000-00005

26. Nicotra A, Asahina M, Young TM, Mathias CJ. Heatprovoked skin vasodilatation in innervated and denervated trunk dermatomes in human spinal cord injury. Spinal Cord. 2006;44(4):222-26. [PMID: 16172627] DOI:10.1038/sj.sc.3101837

27. Schubert V, Fagrell B. Postocclusive reactive hyperemia and thermal response in the skin microcirculation of subjects with spinal cord injury. Scand J Rehabil Med. 1991;23(1): 33-40. [PMID: 2035001]

28. Jan YK, Brienza DM, Boninger ML, Brenes G. Comparison of skin perfusion response with alternating and constant pressures in people with spinal cord injury. Spinal Cord. 2011;49(1):136-41. [PMID: 20514054]

DOI: $10.1038 / \mathrm{sc} .2010 .58$

29. Hagisawa S, Ferguson-Pell M, Cardi M, Miller SD. Assessment of skin blood content and oxygenation in spinal cord injured subjects during reactive hyperemia. J Rehabil Res Dev. 1994;31(1):1-14. [PMID: 8035356]

30. Ping P, Johnson PC. Role of myogenic response in enhancing autoregulation of flow during sympathetic nerve stimulation. Am J Physiol. 1992;263(4 Pt 2):H1177-84. [PMID: 1415767]

31. Powers EW 3rd, Frayer WW. Laser Doppler measurement of blood flow in the microcirculation. Plast Reconstr Surg. 1978;61(2):250-55. [PMID: 622417] DOI:10.1097/00006534-197802000-00016

32. Shepherd AP, Riedel GL, Kiel JW, Haumschild DJ, Maxwell LC. Evaluation of an infrared laser-Doppler blood flowmeter. Am J Physiol. 1987;252(6 Pt 1):G832-39. [PMID: 3591949]

33. Nilsson GE, Tenland T, Oberg PA. Evaluation of a laser Doppler flowmeter for measurement of tissue blood flow. IEEE Trans Biomed Eng. 1980;27(10):597-604. [PMID: 6449469] DOI:10.1109/TBME.1980.326582

34. Fredriksson I, Larsson M, Strömberg T. Measurement depth and volume in laser Doppler flowmetry. Microvasc Res. 2009;78(1):4-13. [PMID: 19285089] DOI:10.1016/j.mvr.2009.02.008

35. Choi CM, Bennett RG. Laser Dopplers to determine cutaneous blood flow. Dermatol Surg. 2003;29(3):272-80.
[PMID: 12614422]

DOI:10.1046/j.1524-4725.2003.29042.x

36. Braverman IM. The cutaneous microcirculation. J Investig Dermatol Symp Proc. 2000;5(1):3-9. [PMID: 11147672] DOI:10.1046/j.1087-0024.2000.00010.x

37. Li Z, Leung JY, Tam EW, Mak AF. Wavelet analysis of skin blood oscillations in persons with spinal cord injury and able-bodied subjects. Arch Phys Med Rehabil. 2006; 87(9):1207-12. [PMID: 16935056]

DOI:10.1016/j.apmr.2006.05.025

38. Beckers F, Verheyden B, Aubert AE. Aging and nonlinear heart rate control in a healthy population. Am J Physiol Heart Circ Physiol. 2006;290(6):H2560-70.

[PMID: 16373585]

DOI:10.1152/ajpheart.00903.2005

39. Liao F, Wang J, He P. Multi-resolution entropy analysis of gait symmetry in neurological degenerative diseases and amyotrophic lateral sclerosis. Med Eng Phys. 2008;30(3): 299-310. [PMID: 17569571]

DOI:10.1016/j.medengphy.2007.04.014

40. Walther C, Gielen S, Hambrecht R. The effect of exercise training on endothelial function in cardiovascular disease in humans. Exerc Sport Sci Rev. 2004;32(4):129-34.

[PMID: 15604930] DOI:10.1097/00003677-200410000-00002

41. Miranda AR, Hassouna HI. Mechanisms of thrombosis in spinal cord injury. Hematol Oncol Clin North Am. 2000; 14(2):401-16. [PMID: 10806563]

DOI:10.1016/S0889-8588(05)70141-6

42. Houtman S, Colier WN, Oeseburg B, Hopman MT. Systemic circulation and cerebral oxygenation during head-up tilt in spinal cord injured individuals. Spinal Cord, 2000; 38(3):158-63. [PMID: 10795936]

DOI:10.1038/sj.sc.3100970

43. Hornyak ME, Naver HK, Rydenhag B, Wallin BG. Sympathetic activity influences the vascular axon reflex in the skin. Acta Physiol Scand. 1990;139(1):77-84. [PMID: 2356759] DOI:10.1111/j.1748-1716.1990.tb08899.x

44. Warner DO, Joyner MJ, Charkoudian N. Nicotine increases initial blood flow responses to local heating of human nonglabrous skin. J Physiol. 2004;559(Pt 3):975-84. [PMID: 15272048]

45. Argacha JF, Adamopoulos D, Gujic M, Fontaine D, Amyai $\mathrm{N}$, Berkenboom G, Van de Borne P. Acute effects of passive smoking on peripheral vascular function. Hypertension. 2008; 51(6):1506-11. [PMID: 18391102] DOI:10.1161/HYPERTENSIONAHA.107.104059

46. Bircher A, De Boer EM, Agner T, Wahlberg JE, Serup J. Guidelines for measurement of cutaneous blood flow by laser Doppler flowmetry. A report from the Standardization Group of the European Society of Contact Dermatitis. Contact 
Dermatitis. 1994;30(2):65-72. [PMID: 8187504] DOI:10.1111/j.1600-0536.1994.tb00565.x

47. Sundberg S. Acute effects and long-term variations in skin blood flow measured with laser Doppler flowmetry. Scand J Clin Lab Invest. 1984;44(4):341-45. [PMID: 6235570] DOI:10.3109/00365518409083817

48. Minson CT, Holowatz LA, Wong BJ, Kenney WL, Wilkins BW. Decreased nitric oxide- and axon reflex-mediated cutaneous vasodilation with age during local heating. J Appl Physiol. 2002;93(5):1644-49. [PMID: 12381749]

Submitted for publication August 6, 2010. Accepted in revised form January 4, 2011.
This article and any supplementary material should be cited as follows:

Liao F, Jan YK. Using multifractal detrended fluctuation analysis to assess sacral skin blood flow oscillations in people with spinal cord injury. J Rehabil Res Dev. 2011;48(7):787-800.

DOI:10.1682/JRRD.2010.08.0145

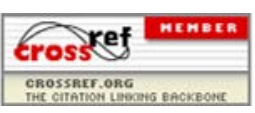


